\title{
Multivariate analysis of inflammatory endotypes in recurrent nasal polyposis in a Chinese population*
}

\author{
Bo Wei', Feng Liu', Jie Zhang²,Yafeng Liu', Jintao Du', Shixi Liu', Nan Zhang³, Rhinology 56: 3, 216-226, 2018 \\ Claus Bachert ${ }^{3,4, \#}$, Juan Meng ${ }^{1 \#}$ \\ https://doi.org/10.4193/Rhin17.240 \\ 'ENT Department, West China Hospital, Sichuan University, Chengdu, China \\ *Received for publication: \\ ${ }^{2}$ The Upper Airway Research Laboratory, West China Hospital, Sichuan University, Chengdu, China \\ December 3, 2017 \\ ${ }^{3}$ Upper Airways Research Laboratory and Department of Oto-Rhino-Laryngology, Ghent University and Ghent University Hospital, Accepted: March 14, 2018 \\ Belgium \\ ${ }^{4}$ Division of ENT Diseases, CLINTEC, Karolinska Institute, University of Stockholm, Sweden \\ \#Equal contribution
}

Background: Chronic rhinosinusitis with nasal polyps (CRSwNP) remains a challenging clinical problem due to its propensity for recurrence. However, data on the frequency of CRSwNP recurrence after surgery in China are rare.

Methods: 78 CRSwNP patients undergoing functional endoscopic sinus surgery were followed-up for 8 years and classified into recurrent and non-recurrent groups. A cluster analysis of the CRSWNP based on inflammatory endotypes was performed, and the endotypes were secondarily matched with clinical phenotypes.

Results: The recurrence rate of CRSwNP in Southwest China was $21.8 \%$ over 8 years post-surgery. The CRSwNP was classified into 4 clusters: cluster 1 (higher expression of IL-5, IgE, and ECP and high positivity rate for SE-IgE); cluster 2 (higher concentrations of IL-6, IL-8 and MPO); cluster 3 (higher concentrations of TNF- $\alpha$ and IFN- $\gamma$ ); and cluster 4 (higher expression of IL-17). Cluster 1 (type-2 inflammation) exhibited the highest recurrence rate, co-morbid asthma and atopy. Notably, the ECP/MPO ratio increased significantly in patients with non-type-2 recurrent CRSwNP 8 years after the first surgery.

Conclusion: Different inflammatory endotypes of CRSwNP exhibited clearly different prognoses. The type-2 subgroup had high recurrence and co-morbid asthma rates comparable to the rates reported in Western countries.

Key words: Recurrent CRSwNP, chronic rhinosinusitis, nasal polyps, cluster analysis, type-2 inflammation

\section{Introduction}

Chronic rhinosinusitis with nasal polyps (CRSwNP) is an inflammatory disease of the nasal and paranasal mucosa that affects $1-4 \%$ of the population ${ }^{(1)}$, and it significantly increases the socioeconomic burden ${ }^{(2,3)}$. CRSwNP remains a challenging clinical problem despite functional endoscopic sinus surgery (FESS) and anti-inflammatory management using corticosteroids and macrolides because of its propensity for recurrence. A report from the United Kingdom showed that $46 \%$ of CRSwNP patients undergo more than one surgery, with a mean number of 3.3 polypectomies ${ }^{(4)}$. Recent retrospective data from China revealed that $55.3 \%$ of CRSwNP patients experienced recurrence within an approximately 34-month follow-up period ${ }^{(5)}$.

CRSWNP is clinically recognized as a subtype of chronic rhinosinusitis (CRS) based on endoscopic tests and/or computerized cosmography ${ }^{(2)}$, and distinctly different inflammatory cell and mediator profiles were found between CRSwNP and chronic rhinosinusitis without nasal polyps (CRSsNP). CRSsNP patients exhibit a predominance of Th1-biased cytokine profiles, and CRSwNP patients exhibited TH2-skewed eosinophilic inflammation in Europe and the United States ${ }^{(6,7)}$. However, CRSwNP is a heterogeneous disease, especially in non-white populations. Neutrophilic inflammation with involvement of Th1 and Th17 cells is also observed ${ }^{(8)}$. The simultaneous expression of different 
Th cell types or the absence of any Th cell types within a single CRSwNP tissue was demonstrated ${ }^{(9)}$. These observations suggest that the classification of CRSWNP is far more complicated than a simple differentiation into Th1 and Th2 subtypes.

Many studies have attempted to identify predictive parameters for CRSwNP recurrence. Chen et al. found that water-cyst type CRSwNP exhibited more and faster recurrence than oedematous granulation type CRSwNP based on endoscopic examination $^{(10)}$. One Japanese study classified CRSwNP into eosinophilic, neutrophilic, and non-eosinophilic non-neutrophilic types according to immunohistochemical analysis, and compared to the other two types, the eosinophilic CRSwNP phenotype is clinically characterized by serum eosinophilia, atopy, extensive disease, and poor prognosis ${ }^{(11)}$. Some studies demonstrated that eosinophil expression in tissue was a prognostic indicator of CRSwNP recurrence. Unfortunately, the precise description and diagnosis for discriminating eosinophilic and non-eosinophilic types remain controversial. The cut-off values varied from 5-350 eosinophils (EOS) per high power field (HPF) ${ }^{(5,12-14)}$, which makes clinical application impossible.

The identification of subgroups is critical because CRSwNP presents a consistent clinical picture but may have variable underlying pathogenesis. Appropriate classification might allow better treatment decision-making and prognostic prediction from an individual perspective. Therefore, this study investigated CRSwNP recurrence in Chinese populations after FESS. We performed cluster analyses of CRSwNP patients based on inflammatory factor endotypes to match these endotypes with clinical phenotypes. This approach could be an applicable classification method for clinical use, could improve the identification of therapeutic targets and could help predict the prognosis of CRSWNP.

\section{Materials and methods}

\section{Patient population}

We prospectively investigated CRSwNP patients who underwent FESS for the first time from June 2008 to April 2009 at the Otolaryngology Department, West China Hospital. The Ethics Committee of West China Hospital of Sichuan University approved this study, and informed consent was obtained from all patients prior to enrolment.

The diagnosis of CRSwNP was based on history, clinical examination, nasal endoscopy and computed tomography (CT) scans of the sinuses as recommended by the European Position Paper on Rhinosinusitis and Nasal Polyps ${ }^{(2)}$, as described previously ${ }^{(15)}$. CT scans were graded according to the Lund-MacKay system, and polyps were graded by size and extent in the left and right nasal fossa on a scale of 0 to 3, according to the classification by Davos $^{(16)}$. Atopy was assessed using a skin prick test for common inhalant allergens. Symptoms were evaluated on a visual analogue scale (VAS) of 0 to 10 . All patients were asked to stop taking antibiotics and oral and topical corticosteroids for at least 1 month prior to surgery. Nasal polyp tissues were obtained during surgery. Samples were snap-frozen in liquid nitrogen upon collection and stored at $-80^{\circ} \mathrm{C}$ until further assessment using immunoassays. Tissue for histochemistry was fixed in $4 \%$ formalin and embedded in paraffin.

All patients had the same post-operative follow-up schedule, which included weekly visits during the first month, monthly visits during the second to third months, and visits every 2 to 3 months until completion of the nasal epithelium repair. All patients were asked to use nasal saline douching and intranasal corticosteroids as ongoing treatment.

All patients were re-examined from October 2016 to March 2017 using nasal endoscopic examination. Tissue was collected if there was nasal polyp recurrence. Symptoms were assessed, and the skin prick test was repeated in all the patients.

\section{Measurement of inflammatory factors}

Samples for inflammatory factor assessment using immunoassays were weighed. A total of $1.0 \mathrm{ml}$ of $0.9 \% \mathrm{NaCl}$ was added per $0.1 \mathrm{~g}$ of each sample, and all samples were homogenized at $1000 \mathrm{rpm}$ for 5 minutes and centrifuged at $1500 \mathrm{~g}$ for 10 minutes at $4^{\circ} \mathrm{C}$. Supernatants were collected and stored at $-20^{\circ} \mathrm{C}$ until analysis. Concentrations of eosinophil cationic protein (ECP), total $\lg \mathrm{E}$, and Staphylococcus aureus enterotoxin-specific lgE (SE-IgE) were assessed using a UniCAP system (Phadia, Uppsala, Sweden). Concentrations of IL-1 $\beta$, IL-5, IL-6, IL-8, IL-17, and TNF- $a$ were assessed using a Luminex 100 system (Luminex, Austin, TX, USA). Concentrations of IFN- $\gamma$ and TGF- $\beta 1$ were assessed using commercially available ELISA kits from R\&D systems (Minneapolis, MN, USA). Myeloperoxidase (MPO) concentrations were measured using a commercially available Myeloperoxidase Human ELISA Kit (ab119605, Abcam, Cambridge, UK). Concentrations in tissue homogenates are expressed as mass versus volume after multiplication by a homogenization dilution factor. Values less than the limit of detection were considered negative for categorical analysis and given a value equal to half of the detection limit for continuous analysis.

\section{Histological evaluation of polyp tissue}

Paraffin-embedded samples were sectioned into $4 \mu \mathrm{m}$-thick slices and stained for MPO (1:200, Abcam, ab9535) and ECP (1:100, Abcam, ab207429). Two independent observers who blinded to the characteristics of the patients assessed the slides. Ten random non-overlapping fields (x400 magnification) were selected, and the results are expressed as the average number of cells per field.

\section{Statistical methods}

IBM SPSS version 24.0.0.0 statistical software was used. A high proportion of values (>33\%) were lower than the detection 
Table 1. Clinical Characteristics of Recurrent versus Non-recurrent CRSwNP patients.

\begin{tabular}{|c|c|c|c|c|c|}
\hline & & Recurrent ( $n=17$ ) & Non-recurrent ( $n=61$ ) & Statistic & $P$ value \\
\hline Total & & $21.8 \%(17 / 78)$ & $78.2 \%(61 / 78)$ & & \\
\hline Age (years) & & $38.7(34.6-43.6)$ & $39.5(29.9-52.4)$ & $Z=-0.272$ & $0.785^{\&}$ \\
\hline \multirow[t]{2}{*}{ Gender } & Male & 13 & 34 & $x 2=1.599$ & $0.206^{€}$ \\
\hline & Female & 4 & 27 & & \\
\hline $\begin{array}{l}\text { History of disease } \\
\text { (months) }\end{array}$ & & $96.0(42.0-120.0)$ & $48.0(24.0-120.0)$ & $Z=-1.222$ & $0.222^{\&}$ \\
\hline Davos score & & $4.0(4.0-6.0)$ & $4.0(2.5-5.0)$ & $Z=-2.052$ & $0.040^{8}$ \\
\hline Lund-Mackay score & & $20.0(10.5-23.0)$ & $14.0(9.5-18.5)$ & $Z=-1.710$ & $0.087^{*}$ \\
\hline \multirow[t]{9}{*}{ VAS } & Severity of the symptom & $6.3(5.5-7.3)$ & $6.0(4.5-7.0)$ & $Z=-0.705$ & $0.481^{*}$ \\
\hline & Nasal blockage & $6.1(3.3-7.2)$ & $5.8(4.6-8.2)$ & $Z=-0.286$ & $0.775^{*}$ \\
\hline & Runny nose & $3.4(0.8-5.3)$ & $4.3(0.5-5.7)$ & $Z=-0.494$ & $0.621^{*}$ \\
\hline & Postnasal drip & $1.2(0.0-2.4)$ & $0.8(0.0-4.5)$ & $Z=-1.036$ & $0.300^{*}$ \\
\hline & Anosmia & $5.2(4.8-9.2)$ & $3.7(0.0-6.6)$ & $Z=-2.199$ & $0.028^{*}$ \\
\hline & Itchy nose & $0.4(0.0-2.6)$ & $0.0(0.0-1.4)$ & $Z=-1.132$ & $0.258^{*}$ \\
\hline & Facial pain & $0.0(0.0-2.2)$ & $0.0(0.0-1.5)$ & $Z=0.000$ & $1.000^{*}$ \\
\hline & Headache & $0.2(0.0-3.3)$ & $3.6(0.0-4.4)$ & $Z=-1.192$ & $0.233^{*}$ \\
\hline & Sneezing & $0.4(0.0-2.8)$ & $0.1(0.0-2.4)$ & $Z=-0.554$ & $0.580^{*}$ \\
\hline \multirow[t]{2}{*}{ Atopy } & Yes & 8 & 20 & $x 2=1.177$ & 0.278 \\
\hline & No & 9 & 41 & & \\
\hline \multirow[t]{2}{*}{ Asthma } & Yes & 5 & 3 & $x^{2}=6.209$ & $0.013^{€}$ \\
\hline & No & 12 & 58 & & \\
\hline \multirow[t]{2}{*}{ Number of smokers } & Yes & 9 & 29 & $x 2=0.155$ & $0.694^{*}$ \\
\hline & No & 8 & 32 & & \\
\hline \multirow[t]{2}{*}{ Number of drinkers } & Yes & 6 & 15 & $x 2=0.774$ & $0.379^{*}$ \\
\hline & No & 11 & 46 & & \\
\hline
\end{tabular}

${ }^{\&}$ Mann-Whitney $\mathrm{U}$ test for non-parametric comparison of two independent samples (median and quartiles). * Pearson chi-squared test, ${ }^{\epsilon}$ Continuity correction chi-squared test.

range and were analysed as binomial variables. The following detection limits were used as cut-offs: IL-5 (cut-off, $6.68 \mathrm{pg} / \mathrm{ml}$ ), SE-lgE (cut-off, $0.35 \mathrm{kU} / \mathrm{L}$ ), TNF-a (cut-off, $16.38 \mathrm{pg} / \mathrm{ml}$ ), IL-17 (cut-off, $11.80 \mathrm{pg} / \mathrm{ml}$ ), and IFN- $\gamma$ (cut-off, $7.8 \mathrm{pg} / \mathrm{ml}$ ).

Baseline parameters were analysed for differences between recurrent and non-recurrent CRSwNP using the Mann-Whitney $\mathrm{U}$ test for continuous variables and the chi-squared test for binomial variables. Inflammatory mediators were also analysed after correction for multiple testing by Holm-Bonferroni adjustment. We used principal component analysis with a correlation matrix after orthogonal rotation with Kaiser normalization to identify relationships between inflammatory factors. Components that explained greater than $70 \%$ of the cumulative information were retained. Variables were analysed using a hierarchical clustering analysis with a cluster method of between-groups linkage and an interval measure of the Pearson correlation. Multidimensional scaling was used with an interval of the Euclidean distance to visualize the subgroups of inflammation factors.

Hierarchical clustering analysis for individual case clustering was used with the cluster method of the between-groups linkage and the interval measure of the squared Euclidean distance, with a range of solutions from two to ten clusters. Fisher's discriminant analysis with leave-one-out classification was used to determine the optimal number of clusters, and the percentage of originally grouped correctly classified cases and the crossvalidated grouped correctly classified cases were calculated. A hierarchical clustering heat map with Multi Experiment Viewer version 4.9.0 (Mev Development Team, https://sourceforge.net/ projects/mev-tm4) was used to describe the characteristics of each cluster concerning inflammatory cytokines, with adjusted data for the median center rows, including hierarchical clustering of Pearson correlations and complete linkage clusting. The Kruskal-Wallis and chi-squared tests were used to compare dissimilarities between clusters because of the non-normal 
Table 2. Inflammatory Mediators in Recurrent versus Non-recurrent CRSwNP patients.

\begin{tabular}{|c|c|c|c|c|c|c|}
\hline & & Recurrent ( $n=14$ ) & Non-recurrent $(n=55)$ & Statistic & $P$ value & $\begin{array}{l}\text { Threshold } \\
\text { after correc- } \\
\text { tion }\end{array}$ \\
\hline $\begin{array}{l}\text { EOS absolute number } \\
\text { in peripheral blood } \\
\left({ }^{*} 10^{9} / \mathrm{L}\right)\end{array}$ & & $0.3(0.2-0.5)$ & $0.1(0.1-0.2)$ & $Z=-3.749$ & $0.000^{*}$ & 0.006 \\
\hline $\begin{array}{l}\text { EOS percent in perip- } \\
\text { heral blood }(\%)\end{array}$ & & $6.9(3.6-8.0)$ & $2.2(1.3-3.4)$ & $Z=-4.026$ & $0.000^{*}$ & 0.006 \\
\hline tlgE in serum (KU/L) & & $131.3(36.8-289.8)$ & $56.0(23.2-113.0)$ & $Z=-1.992$ & $0.046^{*}$ & 0.017 \\
\hline $\begin{array}{l}\text { tlgE in homogenate } \\
(\mathrm{KU} / \mathrm{L})\end{array}$ & & $446.5(117.9-800.5)$ & $67.7(34.7-140.1)$ & $Z=-3.417$ & $0.001^{*}$ & 0.007 \\
\hline IL-6 (pg/ml) & & $131.1(86.9-267.4)$ & $246.1(209.7-676.3)$ & $Z=-2.567$ & $0.010^{*}$ & 0.008 \\
\hline IL-8 (pg/ml) & & $2481.9(936.8-6255.4)$ & $5080.1(2297.6-8273.8)$ & $Z=2.149$ & $0.032^{*}$ & 0.013 \\
\hline MPO (pg/ml) & & $26124.6(6944.1-28561.2)$ & 23506.7 (3461.1-46373.9) & $Z=-0.052$ & $0.958^{*}$ & 0.050 \\
\hline $\mathrm{IL}-1 \beta(\mathrm{pg} / \mathrm{ml})$ & & $28.3(19.2-88.5)$ & $53.0(34.5-139.2)$ & $Z=2.256$ & $0.024^{*}$ & 0.010 \\
\hline $\mathrm{ECP}(\mathrm{pg} / \mathrm{ml})$ & & 9886.5 (1637.5-19773.4) & $1682.2(669.1-3830.8)$ & $Z=3.969$ & $0.000^{*}$ & 0.006 \\
\hline TGF- $\beta 1$ (pg/ml) & & $11428.5(10011.9-14207.6)$ & 11556.9 (7993.9-18406.5) & $Z=-0.179$ & $0.858^{*}$ & 0.025 \\
\hline \multirow[t]{2}{*}{ IL-5 } & Positive & 10 & 11 & $x 2=11.618$ & $0.001^{€}$ & 0.010 \\
\hline & Negative & 4 & 44 & & & \\
\hline \multirow[t]{2}{*}{ SE-lgE } & Positive & 4 & 3 & $x^{2}=4.252$ & $0.039^{€}$ & 0.013 \\
\hline & Negative & 10 & 52 & & & \\
\hline \multirow[t]{2}{*}{ TNF-a } & Positive & 0 & 8 & $x^{2}=1.103$ & $0.294^{€}$ & 0.017 \\
\hline & Negative & 14 & 47 & & & \\
\hline \multirow[t]{2}{*}{ IL-17 } & Positive & 0 & 7 & $x^{2}=0.833$ & $0.362^{€}$ & 0.025 \\
\hline & Negative & 14 & 48 & & & \\
\hline \multirow[t]{2}{*}{ IFN- $\gamma$} & Positive & 2 & 13 & $x^{2}=0.156$ & $0.693^{€}$ & 0.050 \\
\hline & Negative & 12 & 42 & & & \\
\hline
\end{tabular}

Concentrations are expressed as mass per volume of undiluted homogenate. Number of samples showing positivity for the cytokine, as indicated by levels higher than the cut-off value. * Mann-Whitney $U$ test for non-parametric comparison of two independent samples (median and quartiles). $€$ Continuity Correction chi-squared test. Holm-Bonferroni adjustment was used to correct for multiple testing ( $(\mathrm{a}=0.05)$.

distribution of the data and the existence of fewer than 40 cases in each group.

The Wilcoxon signed rank sum test for non-normally distributed data was used to compare the recurrence characteristics of CRSwNP after the first FESS and during follow-up.

\section{Results}

A total of 99 CRSwNP patients underwent surgery from June 2008 to April 2009. Follow-up was performed an average of 8 years after the first surgery, and 21 patients were lost during this time. Seventeen of the remaining 78 patients exhibited recurrence (recurrence rate, $21.8 \%$ ). Two patients in the recurrent group underwent another surgery for nasal polyps during the 8-year period. Seven patients were advised to undergo FESS based on nasal endoscopy (surgical sinus cavity filled with nasal polyps) and severe clinical symptoms. Only 1 patient reported aspirin intolerance.

\section{Clinical characteristics of recurrent versus non-recurrent CRSwNP}

Table 1 (78 patients) summarizes the demographic and clinical characteristics before the first surgery. There was no difference between the two groups in age, gender, disease history, proportion of drinkers and smokers or Lund-Mackay score (CT score). The severity of the symptoms was also not different between these groups, except anosmia, which was more severe in the recurrent group ( $p=0.028)$. Notably, the Davos score was lower in the non-recurrent group $(p=0.040)$. Atopy status was not different between groups, but asthma was more frequent in the recurrent group $(p=0.013)$.

\section{Inflammatory mediators in recurrent versus non-recurrent} CRSwNP

Table 2 (69 samples) shows the expression of total IgE in serum, EOS in peripheral blood, and cytokines in tissue homogenates. 
Table 3. Coordinates from Principal Component Analysis.

\begin{tabular}{|c|c|c|c|c|c|}
\hline & $\begin{array}{l}\text { Compo- } \\
\text { nent } 1\end{array}$ & $\begin{array}{l}\text { Compo- } \\
\text { nent } 2\end{array}$ & $\begin{array}{l}\text { Compo- } \\
\text { nent } 3\end{array}$ & $\begin{array}{c}\text { Compo- } \\
\text { nent } 4\end{array}$ & $\begin{array}{c}\text { Compo- } \\
\text { nent } 5\end{array}$ \\
\hline IL-6 & 0.934 & & & & \\
\hline IL-8 & 0.927 & & & & \\
\hline MPO & 0.807 & & & & \\
\hline IL-5 & & 0.92 & & & \\
\hline $\lg \mathrm{E}$ & & 0.916 & & & \\
\hline ECP & & 0.651 & & & \\
\hline IL-17 & & & 0.766 & & \\
\hline IL-1 $\beta$ & & & 0.652 & & \\
\hline IFN- $\gamma$ & & & & 0.811 & \\
\hline TNF- $a$ & & & & 0.585 & 0.505 \\
\hline \multicolumn{6}{|l|}{ TGF- $\beta 1$} \\
\hline SE-IgE & & & & & 0.899 \\
\hline $\begin{array}{l}\text { Total initial } \\
\text { eigenvalue }\end{array}$ & 2.697 & 2.208 & 1.461 & 1.203 & 1.140 \\
\hline $\begin{array}{l}\text { Variance explained } \\
\text { (\%) }\end{array}$ & 23 & 18 & 12 & 10 & 9 \\
\hline $\begin{array}{l}\text { Cumulative vari- } \\
\text { ance explained (\%) }\end{array}$ & 23 & 41 & 53 & 63 & 72 \\
\hline
\end{tabular}

Variables loading with coordinates less than 0.4 were omitted from the components (the variable loading of TGF- $\beta 1$ was less than 0.4 ), and components with a total initial eigenvalue greater than 1 were retained. The proportion of the total variance in the data explained by each component is given. In addition, the cumulative proportion of the total variance, which is explained by the sum of each of the components and its preceding components, is given. The rotation method was varimax with Kaiser normalization, and the rotation converged in 7 iterations.

The EOS percentage $(p=0.000)$ and absolute numbers $(p=0.000)$ in peripheral blood, total IgE in serum ( $p=0.046)$ and samples $(p=0.001)$, rate of SE-lgE positivity $(p=0.039), E C P(p=0.000)$ and IL-5 $(p=0.001)$ increased in recurrent CRSwNP. In contrast, IL-6 $(p=0.010), I L-8(p=0.032)$, and IL-1 $\beta(p=0.024)$ increased in the non-recurrent group. There was no difference in MPO, TGF- $\beta 1$, TNF- $a$, IL-17, or IFN- $\gamma$ between groups. After Holm-Bonferroni adjustment, tlgE in serum, IL-6, IL-8, IL-1 $\beta$ and rate of SE-lgE positivity were not significantly different between recurrent and non-recurrent CRSwNP anymore.

\section{Cluster analysis of CRSwNP}

Ten of the 78 patients who were followed up did not provide sufficient samples to measure all of the cytokines. Therefore, only 69 patients were enrolled in the cluster analysis.

Principal component analysis (Table 3 ) retained 5 components that explained $72 \%$ of the information of all variables in the data (Kaiser-Meyer-Olkin measure of sampling adequacy equal to 0.613, Bartlett's Test of Sphericity $<0.001$ ). The first component

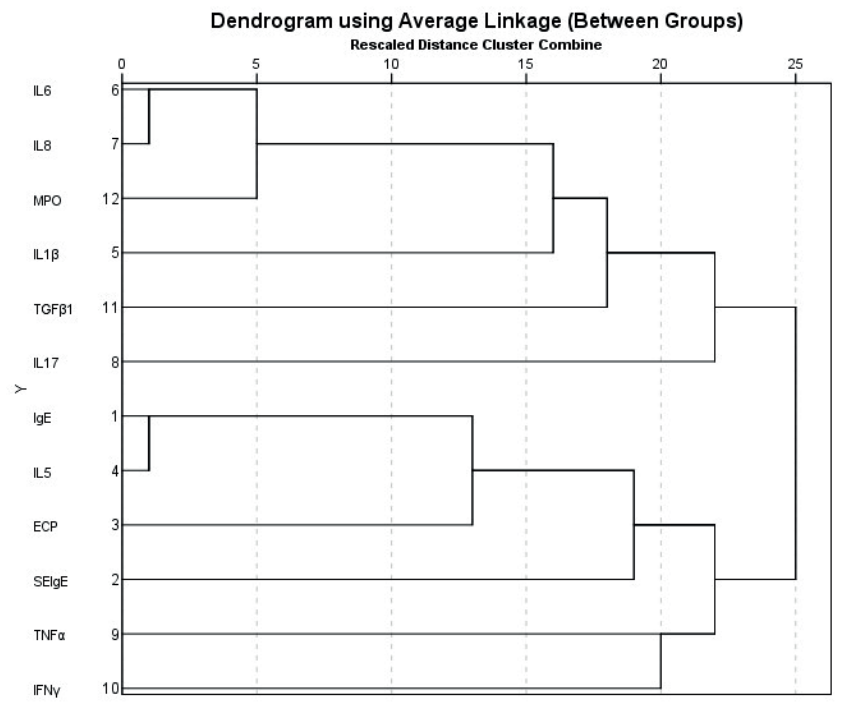

Figure 1. Dendrogram of clustered variables based on hierarchical clustering analysis using a cluster method of between-groups linkage and an interval measure of the Pearson correlation after orthogonal rotation with Kaiser normalization.

consisted of IL-6, IL-8 and MPO. The second component was composed of IL-5, IgE and ECP. The third component included IL17 and IL-1 $\beta$. The fourth component included IFN- $\gamma$ and TNF- $\alpha$, and the last component was composed of SE-IgE and TNF-a. The hierarchical cluster analysis of variables (Figure 1) defined the following optimal clusters: (1) IL-6, IL-8, MPO, IL-1 $\beta$ and TGF- $\beta 1$; (2) IgE, ECP, IL-5, and SE-IgE; (3) IL-17; and (4) TNF- $\alpha$ and IFN- $\gamma$.

Clustering of all CRSWNP patients based on cytokine measurements generated different cluster numbers (ranging from 2 to 10) in the hierarchical cluster analysis. Discriminant analysis with leave-one-out classification was used to validate clustering outcomes. The values of the original and cross-validated correctly classified cases were calculated for each of the possible numbers of clusters (Figure 3). The optimal number of clusters was determined to be 4 because this number appeared at the elbow of the line chart. The clustering of variables was plotted in a 2-dimensional space after multidimensional scaling (Figure 2). Comparison of all cytokine expression levels, except TGF- $\beta 1$ and IL-1 $\beta$, revealed significant differences between the 4 clusters. Cytokines were grouped into the following clusters: (1) IL-5, ECP, IgE, and SE-IgE (type-2 inflammation); (2) MPO, IL-8, and IL-6; (3) TNF- $\alpha$ and IFN- $\gamma$; and (4) IL-17. The expression of IL-5, ECP, IgE, and SE-IgE were significantly higher in cluster 1. MPO, IL-8 and IL-6 were higher in cluster 2 than in the other clusters. TNF- $a$ and IFN- $\gamma$ expression levels were much higher in cluster 3 , and IL-17 was much higher in cluster 4. Hierarchical clustering heat map, which groups each subject based on inflammatory cytokines, indicating four clusters displaying different inflammation patterns as follows: cluster 1 with IL-5, ECP, lgE, and SE-lgE; cluster 2 


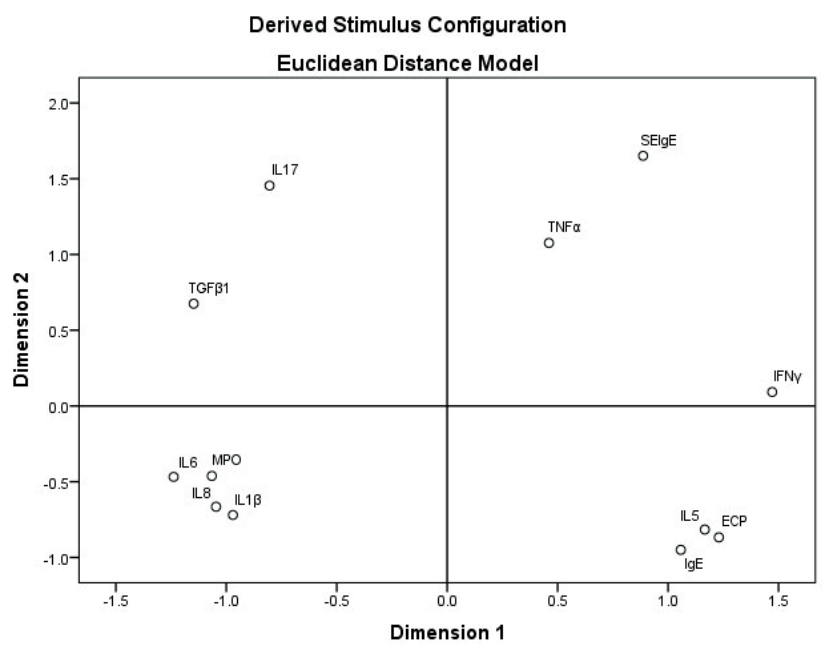

Figure 2. Variables of multidimensional scaling analysis (Repository) Subgroups of inflammatory factors illustrated in a 2-dimensional space using multidimensional scaling. Dissimilarities between all variables of patients were calculated with an interval measure of the Euclidean distance in 2 dimensions. (Badness of fit, stress 0.28299 and goodness of fit, R squared 0.61255).

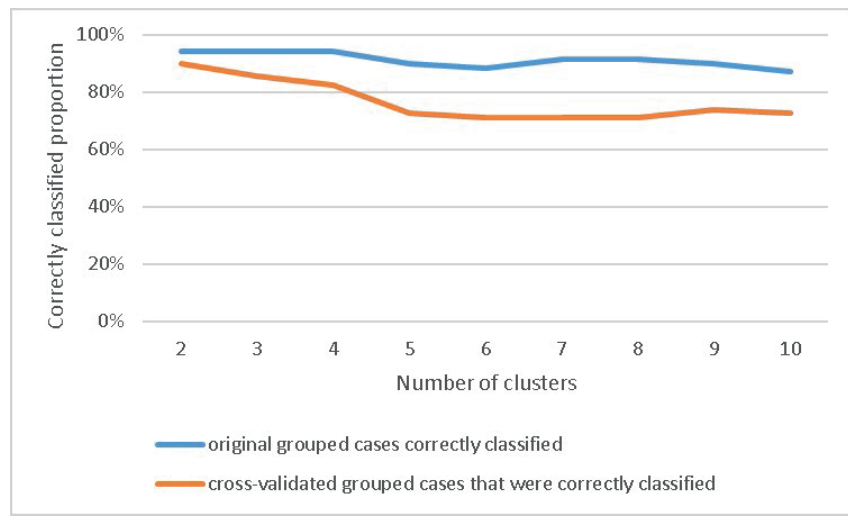

Figure 3. Correctly classified proportion of cluster of discriminant analysis with original and cross-validated grouped cases (Repository). Hierarchical clustering analysis was used with the cluster method of the between-groups linkage and the interval measure of the squared Euclidean distance, with a range of solutions from 2 to 10 clusters. Fisher's discriminant analysis with leave-one-out classification was used to calculate the correctly classified proportion of the original grouped cases and cross-validated grouped cases. with MPO, IL-8, and IL-6; cluster 3 with TNF-a and IFN- ; cluster 4 with IL-17 (Figure 4).

Table 4 and Figure 5 provide the cluster characteristics. Clusters $1,2,3$ and 4 contained $15.9 \%, 37.7 \%, 18.8 \%$, and $27.5 \%$ of the patients, respectively. The recurrence rates of CRSwNP were obviously dissimilar between clusters, with cluster 1 exhibiting the highest recurrence rate $(72.7 \%)$, followed by cluster $2(15.4 \%)$, cluster $3(7.7 \%)$ and cluster 4 (5.3\%). The asthma and atopy status were also different between clusters. Cluster 1 exhibited the highest rates (asthma 36.4\%, atopy $72.7 \%$ ), followed by cluster 2 (asthma 7.7\%, atopy 38.5\%). No patient in cluster 3 had asthma, and $23.1 \%$ of the patients had atopy. In cluster $4,5.3 \%$ of the patients suffered from asthma, and $31.6 \%$ exhibited atopy. Total $\mathrm{IgE}$ in serum and the percentage and absolute number of EOS in peripheral blood were strikingly higher in cluster 1 than in all other clusters. There was no difference in demographic data (age, gender), disease history, Davos and Lund-Mackay scores, symptom severity, or rates of smoking or drinking alcohol between clusters.

\section{Recurrence in non-type-2 CRSwNP}

There were 6 recurrent patients in the non-type-2 CRSwNP group (show in Table 5). The status of allergic rhinitis and asthma did not change for these patients over 8 years. Only one patient switched to SPT positive during the follow-up. However, immunohistochemistry revealed a significantly increased ECP/MPO ratio (median $=7.25$ ) at recurrence compared to that at the first surgery (median $=0.64, p=0.028$ ) 8 years earlier.

\section{Discussion}

We demonstrated that the recurrence rate of CRSwNP was $21.8 \%$ approximately 8 years after surgery in southwest China, which is much lower than the rate in studies from other countries. DeConde reported that the recurrence of CRSwNP in the United States was 35\% six months after FESS, 38\% after 12 months, and $40 \%$ after 18 months ${ }^{(17)}$. Wynn and colleagues found that $60 \%$ of patients developed recurrent polyposis in the United States approximately 40 months after FESS ${ }^{(18)}$. Data from Belgium demonstrated that approximately $80 \%$ of CRSwNP patients exhibited recurrence and approximately $37 \%$ required revision surgery in a prospective follow-up study over 12 years after the initial surgery ${ }^{(19)}$. Our recurrence rate was also much lower than that for northeast part of China, which was $55.3 \%$ approximately 34 months after surgery ${ }^{(5)}$.

Predicting which patients are at higher risk for recurrence remains a challenging clinical problem despite advances in the diagnostic and treatment approaches for CRSwNP because of the complex heterogeneous nature of this disease. Many studies investigated prognostic parameters. Recurrent and nonrecurrent CRSwNP in the present study exhibited no significant differences in clinical characteristics, including CT scans, polyp scores, severity of most symptoms and atopy status, except the asthma rate was higher in the recurrent group. This result is comparable to that of Wynn et al., who also found that patients with asthma are at higher risk of recurrence ${ }^{(18)}$. The role of atopic status in CRSwNP remains controversial based on the limited available data. Some studies demonstrated that nasal polyps with allergy corresponded to a greater recurrence rate ${ }^{(20)}$, but 


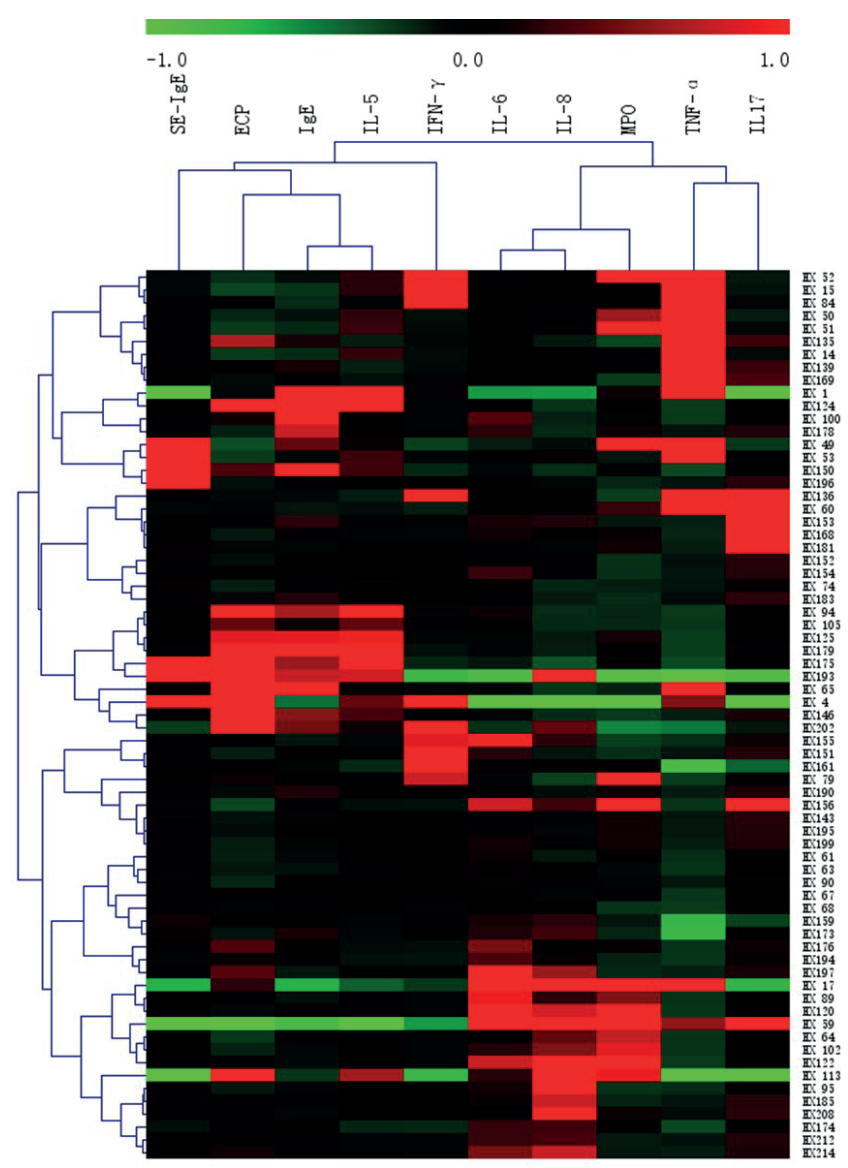

Figure 4. Hierarchical clustering heat map based of inflammatory cytokines. Shown is a hierarchical clustering heat map based on inflammatory cytokines in nasal polyps. Each row represents one subject, and the individual inflammatory cytokines are shown in each column, with green being low and red being high. Left, Dendogram showing similarity of samples. Up, Four clusters are indicated by vertical bars of cluster 1 with IL-5, ECP, IgE, and SE-IgE; cluster 2 with MPO, IL-8, and IL-6; cluster 3 with TNF- $\alpha$ and IFN- $\gamma$; cluster 4 with IL-17.

Erbek and colleagues found no associations between positive skin prick tests and recurrence rate ${ }^{(21)}$. Two recent Chinese studies suggested no association between atopy status and disease severity or recurrence in CRSwNP(22,23). Our study also suggests that routine atopy testing may not be warranted to evaluate the severity and prognosis of CRSWNP(23).

We demonstrated that the recurrent and non-recurrent groups exhibited extremely different inflammatory patterns. The recurrent group expressed a predominantly eosinophilic type of inflammation with higher lgE in serum, higher EOS in peripheral blood and higher IgE, SE-IgE and IL-5 expression in tissue. These findings are consistent with previous data reported by Van Zele et al., who studied caucasian patients and found that non-recurrent CRSwNP patients exhibited significantly higher IFN- $\gamma$ levels and lower concentrations of IgE, ECP, and IL-5 than recurrent CRSwNP patients with a predominant Th2-type of

\begin{tabular}{|c|c|c|c|c|c|c|c|c|}
\hline Cluster & \multicolumn{8}{|c|}{ Inflammatory cy tokine } \\
\hline 1 & $\begin{array}{lll}\text { IL-5 } & \text { ECP } & \text { IgE }\end{array}$ & SE-IgE & & & & & & \\
\hline 2 & & & MPO & IL-8 $\quad$ IL-6 & & & & \\
\hline 3 & & & & & TNF- $\alpha$ & IFN-Y & & \\
\hline 4 & & & & & & & IL-1B & $\mid \mathrm{IL}-17$ \\
\hline
\end{tabular}

A

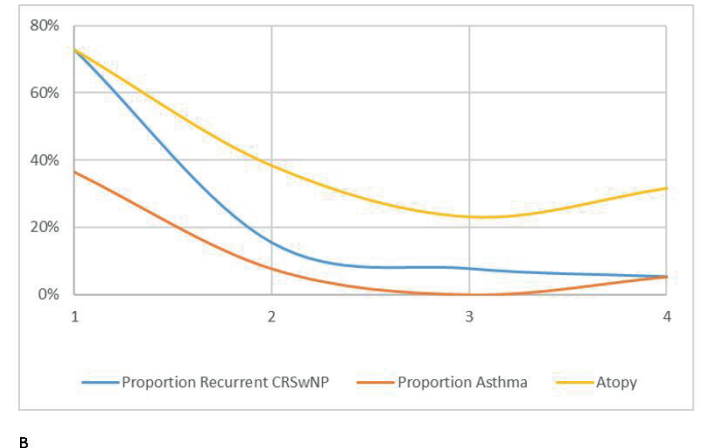

Figure 5. Summary graph. Clusters: 1, Th2 bias group; 2, KCN group; 3 , Th1 bias group; 4, Th17 bias groupA. Simplified graphical representation of the clusters with their specific inflammatory cytokines. For cytokines, white represents no or few increased concentrations, light colours indicate a moderate increase, and dark colours denote a significant increase. B, Line chart showing groups of clusters with inflammatory patterns associated with CRSwNP recurrence, asthma and atopy.

inflammation ${ }^{(6)}$.

An early study by Eweiss demonstrated that the degree of infiltrating eosinophilia in nasal polyps did not differ significantly between recurrent and non-recurrent patients ${ }^{(24)}$. However, more recent evidence demonstrated that CRSwNP with a higher degree of infiltrating eosinophilia exhibited unique characteristics, with greater disease severity and poorer outcome after surgical intervention ${ }^{(5,12,25)}$. Our research also demonstrated that the eosinophilia (ECP IHC) was much higher in the recurrent group. Some studies suggest that CRSwNP should be classified into eosinophilic and non-eosinophilic types according to EOS infiltration. However, the major obstacle to the implementation of this categorization method in clinics is the lack of a standardized definition for eosinophilic CRSwNP. Some studies suggested counting the number of EOS per HPF or calculating the proportion of EOS relative to the rest of the cells. However, the cut-off values varied across studies. Soler et al. demonstrated that $\geq 10$ EOS/HPF as the definition of eosinophil CRS reflected a poor outcome after surgery ${ }^{(26)}$. Studies from Japan demonstrated that defining eosinophilia as $\geq 70$ EOS/HPF identified a significant difference in disease recurrence after endoscopic sinus surgery $^{(11)}$. Lou et al. suggested that an absolute EOS count $>55$ / HPF or a proportion $>27 \%$ of total cells may was an indicator for recurrence ${ }^{(5)}$. Another problem is that the cell distribution in the tissue is uneven, and field selection during cell counting will also interfere with the results.

Therefore, better insight into different inflammatory patterns using cluster analysis might allow the precise identification of 
Table 4. Classifications of the clustering of individual cases.

\begin{tabular}{|c|c|c|c|c|}
\hline & \multicolumn{4}{|c|}{ Cluster } \\
\hline & 1 & 2 & 3 & 4 \\
\hline $\mathrm{N}$ & 11 & 26 & 13 & 19 \\
\hline $\mathrm{N} /$ total & $15.9 \%$ & $37.7 \%$ & $18.8 \%$ & $27.5 \%$ \\
\hline IL-5 (positive rate) & $100 \%$ & $11.5 \%$ & 0 & 0 \\
\hline $\mathrm{ECP}(\mu \mathrm{g} / \mathrm{L})$ & $24425.8(13147.9-38525.4)$ & $3279.7(444.0-3245.7)$ & $3694.9(431.8-3200.6)$ & $2201.9(1105.9-3192.5)$ \\
\hline $\lg E(K U / L)$ & $903.7(428.0-1023.0)$ & $112.5(32.1-126.1)$ & $190.0(10.3-186.2)$ & $152.2(49.5-142.5)$ \\
\hline SE-lgE (positive rate) & $27.27 \%$ & $11.5 \%$ & $7.7 \%$ & 0 \\
\hline MPO (ng/ml) & $25309.5(4992.0-27751.1)$ & 75523.9 (5153.6-137089.9) & 42399.3 (5287.0-85849.6) & 18419.9 (2816.1-27436.2) \\
\hline IL-8 (pg/ml) & $6414.6(771.7-9641.2)$ & $13723.3(2307.2-15596.7)$ & 3951.1 (3108.0-5107.6) & $4896.8(2297.6-7150.0)$ \\
\hline IL-6 (pg/ml) & $238.0(93.3-329.6)$ & $1263.2(166.4-1483.6)$ & $235.1(210.6-246.3)$ & $447.2(151.7-676.3)$ \\
\hline TNF-a (positive rate) & 0 & $3.8 \%$ & $38.5 \%$ & $10.5 \%$ \\
\hline IFN- $\gamma$ (positive rate) & $36.4 \%$ & $15.4 \%$ & $38.5 \%$ & $10.5 \%$ \\
\hline $\mathrm{IL}-1 \beta(\mathrm{pg} / \mathrm{ml})$ & $91.5(10.8-42.1)$ & $97.9(20.0-102.3)$ & $76.3(39.4-95.4)$ & $195.2(52.1-278.5)$ \\
\hline IL-17 (positive rate) & 0 & $7.7 \%$ & 0 & $26.3 \%$ \\
\hline TGF- $\beta 1$ (pg/ml) & $11343.6(8888.0-14188.6)$ & 10839.3 (5624.6-13389.8) & $10744.8(6145.7-14317.0)$ & $20115.2(14388.9-24085.9)$ \\
\hline $\begin{array}{l}\text { Proportion recurrent } \\
\text { CRSwNP }\end{array}$ & $72.7 \%$ & $15.4 \%$ & $7.7 \%$ & $5.3 \%$ \\
\hline Proportion asthma & $36.4 \%$ & $7.7 \%$ & 0 & $5.3 \%$ \\
\hline Proportion atopy & $72.7 \%$ & $38.3 \%$ & $23.1 \%$ & $31.6 \%$ \\
\hline $\begin{array}{l}\text { EOS value in peripheral } \\
\text { blood }(* 109 / \mathrm{L})\end{array}$ & $0.3(0.3-0.4)$ & $0.2(0.1-0.2)$ & $0.2(0.1-0.3)$ & $0.1(0.1-0.2)$ \\
\hline $\begin{array}{l}\text { EOS percent in peripheral } \\
\text { blood }(\%)\end{array}$ & $5.9(3.5-7.5)$ & $3.0(1.4-3.4)$ & $4.3(1.5-5.7)$ & $2.1(1.1-3.3)$ \\
\hline tlgE in serum (KU/L) & $177.1(55.0-264.5)$ & $80.3(11.3-147.4)$ & $96.3(13.6-107.5)$ & $83.6(32.2-111.5)$ \\
\hline
\end{tabular}

Dark green: Concentration significantly higher than 6 or more other clusters; Middle green: Concentration significantly higher than 4 or more other clusters; light green: Concentration significantly higher than 2 or more other clusters.

Columns define clusters of patients with CRSwNP. Rows indicate inflammatory factors used for cluster analysis and clinical parameters. Mean concentrations and quartiles are given for each cluster. Inflammatory variables were handled in the cluster analysis with binomial variables due to a high proportion of measurements lower than the detection limit (IFN- $\gamma$, IL-5, SE-IgE, TNF- $a$, and IL-17). For these variables, the proportions of positive levels are shown. Clinical phenotypes (i.e., proportion with recurrent CRSwNP, asthma and atopy) were tabulated; these phenotypes were not used in cluster analysis. For illustration of the clusters, differentiations of inter-cluster differences are visualized using a colour code as described in the legend.

subgroups for prognosis. This study is the first to present inflammatory endotypes of CRSWNP based on principal component analysis and cluster analysis of biomarkers and demonstrated that 4 groups of cytokines explained the differences among our samples: 1) markers of Th2-skewed eosinophilic inflammation (IL-5, ECP, IgE, and SE-IgE); 2) neutrophilic inflammation markers (MPO, IL-8, and IL-6); 3) Th1 inflammation markers (IFN- $\gamma$ and TNF-a); and 4) Th17 inflammation markers (IL-17). This result is consistent with the Tomassen study in Belgium ${ }^{(27)}$, which also was extended later to Asia and Australia, confirming comparable endotypes, but different prevalences in the different countries $^{(28)}$.

We demonstrated that samples in cluster 1 were all IL-5 positive, and this cluster exhibited the highest rate of SE-IgE and the highest concentrations of ECP and IgE. This group also had the highest rate of co-morbid asthma and atopic status. This cluster clearly demonstrated the characteristics of a Th2-skewed eosinophilic inflammation type of CRSwNP, which is similar to most CRSwNP cases in Western countries. Previous studies demonstrated that $80-85 \%$ of patients with nasal polyps in the United States and Europe exhibited a Th2-biased eosinophilic inflammatory pattern ${ }^{(29)}$. Cluster 1 contained only $15.9 \%$ of all patients in the present study, which is consistent with the results showing that the eosinophilic phenotype constitutes only a small portion of CRSwNP in East Asia, including south China ${ }^{(9,30)}$ and South Korea ${ }^{(31)}$. We demonstrated that the risk of co-morbid asthma was significantly associated with increased expression of IL-5 or total IgE and specific SE-IgE, regardless of ethnicity. 
Table 5. Recurrence in non-type-2 CRSwNP.

\begin{tabular}{|lcccc|}
\hline & $\begin{array}{c}\text { First } \\
\text { surgery }\end{array}$ & Follow-up & Statistics & P value \\
\hline ECP/MPO & $\begin{array}{c}0.64 \\
(0.23-1.63)\end{array}$ & $\begin{array}{c}7.25 \\
(3.51-24.61)\end{array}$ & $\mathrm{Z}=-2.201$ & 0.028 \\
\hline Atopy & 1 & 2 & $\mathrm{Z}=-1.000$ & 0.317 \\
\hline Asthma & 1 & 1 & $\mathrm{Z}=0.000$ & 1.000 \\
\hline
\end{tabular}

Wilcoxon signed rank sum test used to analysis (median and quartiles).

The recurrence rate (21.8\%) of our study was much lower than the figures in Western countries (approximately $57 \%$ over 13 years) ${ }^{(32)}$, but the recurrence rate in cluster 1 was $72.7 \%$, which was obviously much higher than that in the other clusters and similar to that in Western studies. This result verified that cluster 1, which represents the Th2-skewed eosinophilic inflammation type of CRSwNP, represented a form of severe airway inflammation, and the therapeutic approaches used do not lead to longterm symptom control or reduce the risks of recurrence and co-morbid asthma. Therefore, treatments aimed at innovative targets, such as anti-IgE and anti-IL-5 hmAbs, may be considered for this cluster in the future because of the high tissue expression of IgE and IL-5 in this group ${ }^{(29)}$.

Cluster 2 was characterized by higher levels of IL-6, IL-8 and MPO, which is similar to the key cytokine negative (KCN, which means the absence of IL-5, IL-17 and IFN- $\gamma$ expression in tissue) nasal polyps we described previously ${ }^{(9)}$. This cluster was the largest group and contained approximately one-third of the patients. This grouping is consistent with the finding that $28 \%$ of nasal polyps were KCN nasal polyps in Chinese patients compared with only $4 \%$ in Caucasian patients ${ }^{(9)}$. Cluster 3 contained $18.8 \%$ of the patients who were characterized by a higher expression of Th1 cytokines (IFN- $\gamma$ and TNF- $\alpha$ ). Cluster 4 contained $27.5 \%$ of the patients who were characterized by a higher expression of Th17 cytokines (IL-17). Clusters 2, 3, and 4 exhibited very low recurrence rates and low rates of co-morbid asthma. Only 2 patients in cluster 2 were IL-5 positive, and all other patients were IL-5 negative and exhibited a non-eosinophilic inflammatory pattern. We previously found that KCN nasal polyps were associated with a greater gram-negative bacteria load, and IL-5-positive nasal polyps were associated with greater gram-positive bacteria colonization ${ }^{(9)}$. These data suggest that bacterial colonization may impact the inflammatory pattern of nasal polyps. Some studies demonstrated that non-eosinophildominated nasal polyps exhibit a lower response to corticosteroid therapy than the eosinophil-dominated type ${ }^{(33,34)}$. However, long-term low dose macrolide treatment may produce additional anti-inflammatory benefits for neutrophil-associated CRSwNP in East Asian countries ${ }^{(35)}$. The present study suggests that non-eosinophilic inflammation in CRSwNP be further classified into KCN, Th1 and Th17 subgroups. Whether these three subgroups exhibit different underlying pathogenesis and treatment responses to corticosteroids and macrolide therapy is not known and requires further study.

Notably, this study also found increased ECP/MPO and atopic status (number of skin prick test positive) in the tissue of the non-type-2 recurrent CRSwNP patients compared to 8 years before. This suggests that this endotype of CRSwNP might change over time into type-2. This finding is consistent with previous studies in South Korea ${ }^{(36)}$ and Thailand ${ }^{(37)}$, as recently summarized $^{(38)}$ which demonstrated that the inflammatory patterns of CRSwNP in the same geographical area are changing over time and the prevalence of eosinophilic nasal polyps, which were rare in Asians, increased significantly in recent decades. We did not demonstrate an increase in AR and asthma in these patients in the present study. Therefore, the likelihood that they will develop allergic rhinitis and asthma in the future is not known and requires longer follow-up studies to verify.

Our study had several limitations. First, three surgeons in our hospital performed the surgeries on the enrolled patients. The FESS method and treatment strategy after surgery were similar across patients, but we cannot eliminate a bias from the different surgical techniques and therapeutic strategies, which could also affect the prognosis. Second, the regular follow-up stopped when the nasal epithelium repair was complete as determined under endoscopic examination. Therefore, this study could only identify recurrence at a visit long time after surgery, but when recurrence occurred, could not be determined. Third, this study was only a single-centre study with a small sample size. A multicentre study with a larger sample and longer time for regular follow-up is needed in the future. Forth, for recurrent patients, we were able to obtain recurrent nasal polyp tissue biopsies, just enough for IHC. For non-recurrent patients, there was no tissue to be taken, and we did not intend to take normal looking mucosa. Thus, we could not compare cytokine profiles in the non-recurrent or recurrent groups between first surgery and follow-up.

\section{Conclusion}

Despite these shortcomings, this study is the first long-term CRSwNP follow-up study after FESS in a Chinese population. Our findings confirmed that the proportion of the Th2-biased eosinophilic inflammatory pattern was much lower in China than in Western countries. In line with this, the recurrence rate of CRSwNP also was much lower in China; our findings confirmed that different inflammatory endotypes of CRSwNP exhibited completely different prognoses. The type-2 subgroup with high expression of IL-5, ECP, IgE, and SE-IgE exhibited a similar recurrence rate and co-morbid asthma rate as those reported in Western countries ${ }^{(39)}$. KCN, Th1 and Th17 CRSwNP exhibited significantly lower recurrence rates after surgery; however, 
recurrence also in these patients was associated with a shift to type- 2 inflammation, as indicated by an increase in the ECP/ MPO ratio. CRSwNP today is treated as a single clinical entity, but the present study confirms the need for a classification based on immunological endotypes. Endotyping, taking the prognosis of recurrence and asthma comorbidity in account, may benefit the development of personalized treatment strategies for the management of CRSWNP patients.

\section{Acknowledgement}

This work was supported by grants to Juan Meng from the National Science Fund (81300815), the 12th Five-Year Science and Technology Support Program (2014BAI07B04); CB from the European Commission's Seventh Framework programme under grant agreement $N^{\circ} 260895$ (PREDICTA); from the Flemish Scientific Research Board, FWO, projects 1841713N, G.039412N,
G.067512N; BOF14/GOA/019 and the Interuniversity Attraction Poles Program - Belgian State Belgian Science Policy, Nr. IAP $\mathrm{P} 7 / 30$.

\section{Authorship contribution}

Bo Wei and Feng Liu were responsible for the follow-up, data and sample collection and paper writing, Bo Wei and Feng Liu contributed equally to this paper; Jie Zhang was responsible for basic lab examinations; Yafeng Liu, Jintao Du and Shixi Liu were responsible for surgery and follow-up; Nan Zhang was responsible for data analysis; Claus Bachert and Juan Meng were responsible for the whole study design and paper revision.

\section{Conflict of interest}

We declare no potential conflicts of interest involved in this study.

\section{References}

1. Bachert C, Gevaert P, Holtappels G, Cuvelier C, van Cauwenberge P. Nasal polyposis: from cytokines to growth. AmJ Rhinol. 2000;14(5):279-90.

2. Fokkens WJ, Lund VJ, Mullol J, Bachert C, Alobid I, Baroody F, et al. European Position Paper on Rhinosinusitis and Nasal Polyps 2012. Rhinology Supplement. 2012;23:3 p preceding table of contents, 1-298.

3. Bhattacharyya N. Incremental health care utilization and expenditures for chronic rhinosinusitis in the United States. Ann Otol Rhinol Laryngol. 2011;120(7):423-7.

4. Philpott C, Hopkins C, Erskine S, Kumar N, Robertson A, Farboud A, et al. The burden of revision sinonasal surgery in the UK-data from the Chronic Rhinosinusitis Epidemiology Study (CRES): a cross-sectional study. BMJ open. 2015;5(4):e006680.

5. Lou H, Meng Y, Piao Y, Wang C, Zhang L, Bachert C. Predictive significance of tissue eosinophilia for nasal polyp recurrence in the Chinese population. Am J Rhinol Allergy. 2015;29(5):350-6.

6. Van Zele T, Claeys S, Gevaert P, Van Maele G, Holtappels G, Van Cauwenberge P, et al. Differentiation of chronic sinus diseases by measurement of inflammatory mediators Allergy. 2006;61(11):1280-9.

7. Van Bruaene N, Perez-Novo CA, Basinski TM, Van Zele T, Holtappels G, De Ruyck N, et al. T-cell regulation in chronic paranasal sinus disease. J Allergy Clin Immunol. 2008;121(6):1435-41, 41 e1-3.

8. Zhang N, Van Zele T, Perez-Novo C, Van Bruaene N, Holtappels G, DeRuyck N, et al. Different types of T-effector cells orchestrate mucosal inflammation in chronic sinus disease. J Allergy Clin Immunol. 2008;122(5):961-8.

9. Ba L, Zhang N, Meng J, Zhang J, Lin P, Zhou $P$, et al. The association between bacterial colonization and inflammatory pattern in
Chinese chronic rhinosinusitis patients with nasal polyps. Allergy. 2011;66(10):1296-303.

10. Chen W, Sun Q, Deng Y. [Clinical and pathological observation of recurrent nasal polyp formation]. Zhonghua er bi yan hou ke za zhi. 1997;32(1):15-7.

11. Ikeda K, Shiozawa A, Ono N, Kusunoki T, Hirotsu M, Homma H, et al. Subclassification of chronic rhinosinusitis with nasal polyp based on eosinophil and neutrophil. Laryngoscope. 2013;123(11):E1-9.

12. Soler ZM, Sauer DA, Mace J, Smith TL. Relationship between clinical measures and histopathologic findings in chronic rhinosinusitis. Otolaryngol Head Neck Surg 2009:141(4):454-61.

13. Nakayama T, Yoshikawa M, Asaka D, Okush T, Matsuwaki Y, Otori N, et al. Mucosal eosinophilia and recurrence of nasal polyps new classification of chronic rhinosinusitis. Rhinology. 2011;49(4):392-6.

14. Yao T, Kojima Y, Koyanagi A, Yokoi H, Saito T, Kawano K, et al. Eotaxin-1, -2, and -3 immu noreactivity and protein concentration in the nasal polyps of eosinophilic chronic rhinosinusitis patients. Laryngoscope. 2009;119(6):1053-9.

15. Li X, Meng J, Qiao X, Liu Y, Liu F, Zhang N, et al. Expression of TGF, matrix metalloproteinases, and tissue inhibitors in Chinese chronic rhinosinusitis. J Allergy Clin Immunol. 2010;125(5):1061-8.

16. Fokkens $W$, Lund $V$, Bachert C, Clement $P$, Helllings $P$, Holmstrom $M$, et al. EAACl position paper on rhinosinusitis and nasal polyps executive summary. Allergy. 2005;60(5):583-601.

17. DeConde AS, Mace JC, Levy JM, Rudmik L, Alt JA, Smith TL. Prevalence of polyp recurrence after endoscopic sinus surgery for chronic rhinosinusitis with nasal polyposis. Laryngoscope. 2017;127(3):550-5.

18. Wynn R, Har-El G. Recurrence rates after endoscopic sinus surgery for massive sinus polyposis. Laryngoscope. 2004;114(5):811-3.

19. Gevaert P, Calus L, van Bruaene N, Van Zele T, Bachert C. Allergic Sensitization, High Local IL-5 and lgE Predict Surgical Outcome 12 Years after Endoscopic Sinus Surgery for Chronic Rhinosinusitis with Nasal Polyposis. J Allergy Clin Immunol 2015;135(2):Ab238$\mathrm{Ab}$

20. Dursun E, Korkmaz H, Eryilmaz A, Bayiz U, Sertkaya D, Samim E. Clinical predictors of long-term success after endoscopic sinus surgery. Otolaryngol Head Neck Surg. 2003;129(5):526-31.

21. Erbek SS, Erbek S, Topal O, Cakmak O. The role of allergy in the severity of nasal polyposis. Am J Rhinol. 2007;21(6):686-90.

22. Lou H, Meng Y, Piao $Y$, Zhang N, Bachert C, Wang $C$, et al. Cellular phenotyping of chronic rhinosinusitis with nasal polyps. Rhinology. 2016;54(2):150-9.

23. Li QC, Cheng KJ, Wang F, Zhou SH. Role of atopy in chronic rhinosinusitis with nasal polyps: does an atopic condition affect the severity and recurrence of disease? J Laryngol Otol. 2016;130(7):640-4.

24. Eweiss A, Dogheim $Y$, Hassab M, Tayel $H_{\text {, }}$ Hammad Z. VCAM-1 and eosinophilia in diffuse sino-nasal polyps. Eur Arch Otorhinolaryngol. 2009;266(3):377-83.

25. Tokunaga T, Sakashita M, Haruna T, Asaka D, Takeno S, Ikeda $H$, et al. Novel scoring system and algorithm for classifying chronic rhinosinusitis: the JESREC Study. Allergy. 2015;70(8):995-1003.

26. Soler ZM, Sauer D, Mace J, Smith TL. Impact of mucosal eosinophilia and nasal polyposis on quality-of-life outcomes after sinus surgery. Otolaryngol Head Neck Surg. 2010;142(1):64-71.

27. Tomassen P, Vandeplas G, Van Zele T, Cardell $\mathrm{LO}$, Arebro J, Olze $\mathrm{H}$, et al. Inflammatory endotypes of chronic rhinosinusitis based on cluster analysis of biomarkers. J Allergy Clin Immunol. 2016;137(5):1449-56 e4. 
28. Wang $X$, Zhang N, Bo M, Holtappels G Zheng $M$, Zhang L, et al. Diversity of T helper cytokine profiles in chronic rhinosinusitis: a multicenter study in Europe, Asia and Oceania. Allergy. 2016;71:13-.

29. Bachert C, Zhang L, Gevaert P. Current and future treatment options for adult chronic rhinosinusitis: Focus on nasal polyposis. Allergy Clin Immunol. 2015;136(6):1431-40; quiz 41.

30. Cao PP, Li HB, Wang BF, Wang SB, You XJ, Cui $\mathrm{YH}$, et al. Distinct immunopathologic characteristics of various types of chronic rhinosinusitis in adult Chinese. J Allergy Clin Immunol. 2009;124(3):478-84, 84 e1-2.

31. Kim JW, Hong SL, Kim YK, Lee CH, Min YG, Rhee CS. Histological and immunological features of non-eosinophilic nasal polyps. Otolaryngol Head Neck Surg. 2007;137(6):925-30

32. Philpott C, Philpott C, Erskine S, Kumar N, Robertson A, Farboud A, et al. The burden of revision sinonasal surgery in the UK-data from the Chronic Rhinosinusitis Epidemiology Study (CRES): a cross-sectional study. BMJ Open. 2015;5(4).

33. Wen W, Liu W, Zhang L, Bai J, Fan Y, Xia W, et al. Increased neutrophilia in nasal polyps reduces the response to oral corti costeroid therapy. J Allergy Clin Immunol.
2012;129(6):1522-8 e5.

34. Kirtsreesakul V, Atchariyasathian V. Nasal polyposis: role of allergy on therapeutic response of eosinophil- and noneosinophil-dominated inflammation. Am J Rhinol. 2006;20(1):95-100

35. Shimizu T, Suzaki H. Past, present and future of macrolide therapy for chronic rhinosinusitis in Japan. Auris, nasus, larynx. 2016:43(2):131-6.

36. Kim SJ, Lee KH, Kim SW, Cho JS, Park YK, Shin SY. Changes in histological features of nasal polyps in a Korean population over a 17-year period. tolaryngol Head Neck Surg. 2013;149(3):431-7.

37. Katotomichelakis M, Tantilipikorn $P$, Holtappels G, De Ruyck N, Feng L, Van Zele $T$, et al. Inflammatory patterns in upper airway disease in the same geographical area may change over time. Am J Rhinol Allergy. 2013;27(5):354-60

38. Zhang Y, Gevaert E, Lou HF, Wang XD, Zhang L, Bachert $C$, et al. Chronic rhinosinusitis in Asia. J Allergy Clin Immunol. 2017;140(5):1230-9.

39. Van Zele T, Holtappels G, Gevaert P, Bachert C. Differences in initial immunoprofiles between recurrent and nonrecurrent chronic rhinosinusitis with nasal polyps. Am J Rhinol Allergy. 2014;28(3):192-8.

\section{Juan Meng}

ENT Department

West China Hospital

Sichuan University

No.37 Guoxue Alley

610041 Chengdu, Sichuan

China

E-mail:mjmelinda@163.com

Prof. Claus Bachert

Upper Airways Research Laboratory

ENT Department

Ghent University

De Pintelaan 185

9000 Ghent

Belgium

E-mail: claus.bachert@ugent.be 